\title{
Measuring Smoothness of Trigonometric Interpolation Through Incomplete Sample Points
}

\author{
Stephan Weiss ${ }^{1}$, Jesus Selva ${ }^{2}$, and Malcolm D. Macleod ${ }^{1,3}$ \\ ${ }^{1}$ Department of Electronic \& Electrical Engineering, University of Strathclyde, Glasgow, Scotland \\ ${ }^{2}$ Signals, Systems and Telecommunications Group, University of Alicante, Alicante, Spain \\ ${ }^{3}$ QinetiQ Ltd., Malvern, UK \\ stephan.weiss@strath.ac.uk, jesus.selva@ua.es,mmacleod@qinetiq.com
}

\begin{abstract}
In this paper we present a metric to assess the smoothness of a trigonometric interpolation through an incomplete set of sample points. We measure smoothness as the power of a particular derivative of a $2 \pi$-periodic Dirichlet interpolant through some sample points. We show that we do not need to explicitly complete the sample set or perform the interpolation, but can simply work with the available sample points, under the assumption that any missing points are chosen to minimise the metric, and present a simple and robust approach to the computation of this metric. We assess the accuracy and computational complexity of this approach, and compare it to benchmarks.
\end{abstract}

\section{INTRODUCTION}

In the context of broadband array processing, the eigenvalue decomposition of a parahermitian polynomial matrix $\boldsymbol{R}(z)$ [1] has in most cases the factorisation into analytic eigenvalues and eigenvectors [2], [3] as a desirable solution. Approximating this solution requires a metric that distinguishes this solution from others, which approximate non-differentiable or even discontinuous functions as obtained by many existing algorithms [1], [4], [5]. An approximation of this analytic solution is advantageous as it minimises the order - and therefore the associated computational complexity [6] — of the factors that enable solutions to problems such as source separation [7], angle of arrival estimation [8], broadband beamforming [9], and many more.

Similar challenges to identify an analytic and therefore infinitely differentiable and smooth function have arisen, for example, in the analytic singular value decomposition [10][15]. There metrics such as minimum arc length have been employed in [11] in order to determine the analytic solution. Different from [10]-[15], we specifically operate in a discrete time scenario, where $\boldsymbol{R}(z)$, when evaluated in the unit circle

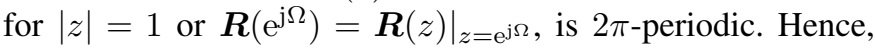
we are also looking for an analytic solution that reflects this $2 \pi$-periodicity.

To extract analytic eigenvalues, new polynomial EVD algorithms operate in the discrete Fourier transform (DFT) domain, and the challenge there is to associate functions smoothly across independent frequency bins [16]. Previously, the power in a derivative of a Dirichlet or trigonometric interpolation has

This work was in parts supported by the Engineering and Physical Sciences Research Council (EPSRC) Grant number EP/S000631/1 and the MOD University Defence Research Collaboration in Signal Processing. been proven as a powerful metric for this. However, for $M$ eigenvalues across $K$ frequency bins, in principle, $(M)^{K-1}$ different associations are possible, which is not feasible even for moderate values of $M$ and $K$. Therefore, in [16] a maximum likelihood sequence estimation approach inspects possible associations as the number of considered sample points iteratively increases from $J=2$ to $J=K$. This drastically reduces the search space, but requires the interpolation from a only a subset of $J<K$ sample points on the unit circle, similar to the 'missing samples problem' [17]. Any yet unassigned sample points are chosen such that a maximally smooth function for the given sample set is extracted. As a drawback, the calculation of this metric in [18], [19] is both computationally costly and can be poorly conditioned.

Therefore in this paper, we propose a calculation of the smoothness metric in [18], [19] that bypasses the inversion of an ill-conditioned matrix for every value of $J$ and $K$, with a QR decomposition [20] that is independent of $J$ and only needs to be evaluated once for a value of $K$. Thus, the evaluation of the metric is better conditioned (thus avoiding biased results due to regularisation necessary in [18], [19]) and overall significantly less costly.

In order to present the approach method, Sec. II defines the Dirichlet or trigonometric interpolation of sample points of $2 \pi$ periodic functions. Sec. III characterises the missing samples problem, and reviews a Schur complement approach [18], [19], which in Sec. IV forms the basis of the proposed method. We present numerical examples and a comparison in Sec. V and draw conclusions in Sec. VI.

\section{MaXimally Smooth Interpolation}

Before we address the incomplete sample set or missing samples problem in a subsequent section, we explore the issue of interpolation given a complete, regularly spaced set of samples, $F_{k}=F\left(\mathrm{e}^{\mathrm{j} \Omega_{k}}\right)$ with $\Omega_{k}=2 \pi k / K, k=0, \ldots(K-1)$, and how we can measure the smoothness of the interpolant.

\section{A. Dirichlet Interpolation}

We based an interpolant $\hat{F}\left(\mathrm{e}^{\mathrm{j} \Omega}\right)$ on an interpolation function $P_{K}\left(\mathrm{e}^{\mathrm{j} \Omega}\right)$, such that

$$
\hat{F}\left(\mathrm{e}^{\mathrm{j} \Omega}\right)=\frac{1}{K} \sum_{k=0}^{K-1} F_{k} P_{K}\left(\mathrm{e}^{\mathrm{j}\left(\Omega-\Omega_{k}\right)}\right) .
$$


In this case, a Dirichlet kernel can provide a maximally smooth interpolation in the sense that the time domain support of $\hat{f}[n] \circ-\bullet \hat{F}\left(\mathrm{e}^{\mathrm{j} \Omega}\right)$ will be as short as possible. For reasons that will be addressed below, we must distinguish between a complex-valued and real valued interpolation, and, for the latter, between odd and even support $K$ of this kernel $P_{K}\left(\mathrm{e}^{\mathrm{j} \Omega}\right)$.

In the case of a complex-valued interpolation, we assume that $\hat{f}[n]$ is causal and that the time domain equivalent of the Dirichlet kernel forms a rectangular window starting from $n=0$,

$$
p_{K}[n]= \begin{cases}1, & 0 \leq n<K \\ 0, & \text { otherwise } .\end{cases}
$$

The Dirichlet kernel or periodic sinc function $P_{k}\left(\mathrm{e}^{\mathrm{j} \Omega}\right)$,

$$
P_{K}\left(\mathrm{e}^{\mathrm{j} \Omega}\right)=\sum_{k=0}^{K-1} \mathrm{e}^{-\mathrm{j} k \Omega}=\mathrm{e}^{-\mathrm{j} \frac{K-1}{2} \Omega} \frac{\sin \left(\frac{K}{2} \Omega\right)}{\sin \left(\frac{1}{2} \Omega\right)},
$$

is well-established in the signal processing community [21], [22].

For the case where $f_{k}$ and the interpolant $\hat{F}\left(\mathrm{e}^{\mathrm{j} \Omega}\right)$ are realvalued, $f[n]$ necessarily is symmetric. This property must be reflected by the kernel. For odd values of $K$, it is straightforward to achieve this by shifting $p_{k}^{(\text {r,odd })}[n]=p_{K}\left[n-\frac{K-1}{2}\right]$ to sit symmetrically w.r.t. $n=0$, and thus creating a zero-phase

$$
\left.P^{(\mathrm{r}, \text { odd }}\right)_{K}\left(\mathrm{e}^{\mathrm{j} \Omega}\right)=\frac{\sin \left(\frac{K}{2} \Omega\right)}{\sin \left(\frac{1}{2} \Omega\right)} .
$$

For even values of $K, p_{k}[n]$ in its above definition cannot be centred on the sampling grid. Likewise, omitting the phase term from $P_{K}\left(\mathrm{e}^{\mathrm{j} \Omega}\right)$ leads to a $4 \pi$-periodic function, losing the demanded $2 \pi$-periodicity. Hence the requirement of a maximally short but symmetric time domain equivalent leads to $[23]$

$$
p_{K}^{(\mathrm{r}, \text { even })}[n]=\frac{1}{2}\left(p_{K}\left[n-\frac{K}{2}\right]+p_{K}\left[n-\frac{K}{2}+1\right]\right),
$$

or equivalently

$$
P_{K}^{(\mathrm{r}, \text { even })}\left(\mathrm{e}^{\mathrm{j} \Omega}\right)=\frac{\sin \left(\frac{K}{2} \Omega\right)}{\tan \left(\frac{1}{2} \Omega\right)}
$$

for the real-valued Dirichlet kernel for $K$ even.

In all cases, the Dirichlet kernel can we expressed as a sum of complex exponentials,

$$
\sum_{k=0}^{K-1} \mathrm{e}^{-\mathrm{j}(k-L) \Omega}=\sum_{n=-L}^{K-L-1} \mathrm{e}^{-\mathrm{j} n \Omega}=\sum_{n=0}^{K-1} \mathrm{e}^{-\mathrm{j} n \Omega},
$$

with generally $L=0$ for the complex valued case. For the real-valued case, odd $K$ means $L=\frac{K-1}{2}$, while for $K$ even, $L=\frac{K}{2}-1$. For the latter, the support of $p_{K}^{(\mathrm{r}, \text { even })}[n]$ is $K+1$, but the outer complex exponential terms in the expansion of(6), weighted by $\frac{1}{2}$ each, are aliased versions of each other, and can be combined with a unit weight. However, due to aliasing the sum can be simplified without any offset as in the last step of (7).

\section{B. Smoothness Metric}

To determine the smoothness of the interpolant $\hat{F}\left(\mathrm{e}^{\mathrm{j} \Omega}\right)$, we rely on the power in its $p$ th derivative [18], [19],

$$
\chi_{p}=\frac{1}{2 \pi} \int_{-\pi}^{\pi}\left|\frac{\mathrm{d}^{p}}{\mathrm{~d} \Omega^{p}} \hat{F}\left(\mathrm{e}^{\mathrm{j} \Omega}\right)\right|^{2} \mathrm{~d} \Omega
$$

For $\hat{F}\left(\mathrm{e}^{\mathrm{j} \Omega}\right)$, with (7) we can write

$$
\begin{aligned}
\hat{F}\left(\mathrm{e}^{\mathrm{j} \Omega}\right) & =\frac{1}{K} \sum_{k=0}^{K-1} F_{k} \sum_{n=0}^{K-1} \mathrm{e}^{-\mathrm{j} n\left(\Omega-\Omega_{k}\right)} \\
& =\sum_{n=0}^{K-1} \hat{f}[n] \mathrm{e}^{-\mathrm{j} n \Omega}=\sum_{n=-L}^{K-L-1} \hat{f}[n] \mathrm{e}^{-\mathrm{j} n \Omega},
\end{aligned}
$$

as expected for the inverse Fourier transform. For the last step in (10), we have exploited the discrete Fourier transform applied to $F_{k}$. Because $F_{k}$ is discrete and periodic, so is $\hat{f}[n]$. Hence, we focus on a single period around zero; it no longer matters whether $F_{k}$ is real-valued or complex valued, but for both cases we distinguish between $L=(K-1) / 2$ of $K$ odd, and $L=K / 2-1$ for $K$ even.

Since $\hat{f}[n] \circ-F_{k}$, the differentiation property of the Fourier transform or direct consideration of (10) lead to the $p$ th derivative of $\hat{F}\left(\mathrm{e}^{\mathrm{j} \Omega}\right)$ w.r.t. the normalised angular frequency $\Omega$,

$$
\frac{\mathrm{d}^{p}}{\mathrm{~d} \Omega^{p}} \hat{F}\left(\mathrm{e}^{\mathrm{j} \Omega}\right)=\sum_{n=-L}^{K-L-1}(-\mathrm{j} n)^{p} \hat{f}[n] \mathrm{e}^{-\mathrm{j} n \Omega} .
$$

Therefore using Parseval's theorem [22], we obtain

$$
\chi_{p}=\sum_{n=-L}^{K-L-1} n^{2 p}|\hat{f}[n]|^{2}=\frac{1}{K^{2}} \mathbf{f}^{\mathrm{H}} \mathbf{W} \mathbf{D}_{K, L, p}^{2} \mathbf{W}^{\mathrm{H}} \mathbf{f},
$$

whereby $\hat{f}[n]$ is related back to the sample points $\mathbf{f}=$ $\left[F_{0}, \ldots, F_{K-1}\right]^{\mathrm{T}}, \mathbf{W}$ via a $K$-point DFT-matrix. Because the DFT matrix operated from index $k=0$ to $K-1$, the order of the coefficients $(\mathrm{j} n)^{p}$ has to be reflected in the diagonal weighting matrix

$$
\mathbf{D}_{K, L, p}=\operatorname{diag}\left\{0, \ldots,(K-L-1)^{p},(-L)^{p}, \ldots,(-1)^{p}\right\} .
$$

Similar to [18], [19], it is also possible to accumulate derivative powers up to the $P$ th order, such that $\chi^{(P)}=\sum_{p=0}^{P} \chi_{p}=$ $\mathbf{f}^{\mathrm{H}} \sum_{p=0}^{P} \mathbf{D}_{K, L, p}^{2} \mathbf{f}$.

\section{Properties of the 'Smoothness Matrix'}

The matrix $\mathbf{C}=\mathbf{W D}_{K, L, p}^{2} \mathbf{W}^{\mathrm{H}}$ that weighs the inner product in (12) has been referred to as a 'smoothness matrix' [18] since it is central to measuring the smoothness $\mathbf{f}^{\mathrm{H}} \mathbf{C f}$ of a Dirichlet interpolation through the elements of $\mathbf{f}$. We note that the elements $d[n]$ along the diagonal of $\mathbf{D}_{K, L, p}^{2}$ are real valued by construction and symmetric by inspection of (13). Since $\mathbf{W}$ is a discrete Fourier transform (DFT) matrix, we find that (i) the elements $d[n]$ are the eigenvalues of $\mathbf{C}$, and (ii) that $\mathbf{C}$ is a circulant matrix. 
The elements of the the circulant matrix $\mathbf{C}$ are made up of the DFT of $d[n]$. Since $d[n]$ is real-valued and symmetric, its DFT will be symmetric and real-valued. Hence, $\mathbf{C}(z)$ is real-valued, $\mathbf{C} \in \mathbb{R}^{K \times K}$. Since its eigenvalues satisfy $d[n] \geq$ 0 , it also is a positive semi-definite matrix. From the above definition of smoothness, $\chi_{p}$, there is at least one eigenvalue that is zero. The remaining eigenvalues, $n^{2 p}$, can possess a considerable dynamic range.

\section{Missing SAMPLES PROBlem}

\section{A. Optimisation Problem}

In a missing samples problem [17], some of sample points $F_{k}, k=0, \ldots(K-1)$ are unavailable, and the aim is to replace these missing sample points such that the interpolation through the given ones is as smooth as possible. Here, we define smoothness in the sense of the metric $\chi_{p}$ in (8), and partition the vector $\mathbf{f}=\left[F_{0}, \ldots, F_{K-1}\right]^{\mathrm{T}}$ as $\mathbf{f}=\left[\mathbf{f}_{J \mid K}^{\mathrm{T}} \mathbf{x}^{\mathrm{T}}\right]^{\mathrm{T}}$, where $\mathbf{f}_{J \mid K} \in \mathbb{C}^{J}, J<K$ contains the given samples, and $\mathbf{x} \in \mathbb{C}^{K-J}$ the missing sample points. Thus the determination of a smoothness metric for the missing samples problem can be formulated as

$$
\chi_{p}=\min _{\mathbf{x}}\left[\mathbf{f}_{J \mid K}^{\mathrm{H}} \mathbf{x}^{\mathrm{H}}\right] \mathbf{C}\left[\begin{array}{c}
\mathbf{f}_{J \mid K} \\
\mathbf{x}
\end{array}\right],
$$

where $\mathbf{C}=\mathbf{W} \mathbf{D}_{K, L, p}^{2} \mathbf{W}^{\mathrm{H}}$ as defined in (12).

For simplicity, (14) assumes that the given samples are contiguous with $\mathbf{f}_{J \mid K}=\left[\begin{array}{lll}F_{0}, \ldots, & F_{J-1}\end{array}\right]^{\mathrm{T}}$. However, an arbitrary arrangement is possible by absorbing a permutation matrix into $\mathbf{C}$. Similarly, the problem can accommodate a cumulative cost $\chi^{(P)}$ by changing the diagonal component of C. To address the resulting problem, [18], [19] have explored a constrained optimisation problem and a Schur-based approach. We below briefly sketch the Schur-based approach, which will form the basis of what Sec. IV will propose.

\section{B. Schur Complement-Based Solution}

To solve (14), we partition $\mathbf{C}$ into

$$
\mathbf{C}=\left[\begin{array}{ll}
\mathbf{C}_{1} & \mathbf{C}_{2} \\
\mathbf{C}_{2}^{\mathrm{T}} & \mathbf{C}_{4}
\end{array}\right]
$$

where $\mathbf{C}_{1} \in \mathbb{R}^{J \times J}, \mathbf{C}_{2} \in \mathbb{R}^{J \times K}$, and $\mathbf{C}_{4} \in \mathbb{R}^{K \times K}$. Subsequently, the cost function becomes

$$
\begin{aligned}
\mathbf{f}^{\mathrm{H}} \mathbf{C f}=\mathbf{f}_{J \mid K}^{\mathrm{H}} \mathbf{C}_{1} \mathbf{f}_{J \mid K}+\mathbf{f}_{J \mid K}^{\mathrm{H}} \mathbf{C}_{2} \mathbf{x}+ \\
+\mathbf{x C}_{2}^{\mathrm{T}} \mathbf{f}_{J \mid K}+\mathbf{f}_{J \mid K}^{\mathrm{H}} \mathbf{C}_{4} \mathbf{f}_{J \mid K} .
\end{aligned}
$$

By addressing this quadratic problem using Wirtinger calculus [24] to differentiate (16) w.r.t. $\mathbf{x}$ and setting the gradient to zero, we obtain the least squares solution $\mathbf{x}_{\mathrm{opt}}=-\mathbf{C}_{4} \mathbf{C}_{2}^{\mathrm{T}} \mathbf{f}_{J \mid K}$ for the optimal completion vector, as well as

$$
\chi_{p}=\mathbf{f}_{J \mid K}^{\mathrm{H}}\left(\mathbf{C}_{1}-\mathbf{C}_{2} \mathbf{C}_{4}^{-1} \mathbf{C}_{2}^{\mathrm{T}}\right) \mathbf{f}_{J \mid K}
$$

for the minimum mean square error. The term $\mathbf{C}_{1}-\mathbf{C}_{2} \mathbf{C}_{4}^{-1} \mathbf{C}_{2}^{\mathrm{T}}$ is known as the Schur complement of $\mathbf{C}$ [20].

The solution in (17) is problematic, since $\mathbf{C}$ is rank deficient, and even a subpartition of it, because of its eigenvalues $n^{2 p}$, is likely ill-conditioned. Particularly for $J \longrightarrow K$, (i) the cost for the inversion of $\mathbf{C}_{4}$ increases and (ii) its conditioning worsens [18]. An alternative is a constrained optimisation approach to solve for $\mathbf{x}_{\mathrm{opt}}$ and $\chi_{p}$, which behaves better for $J \longrightarrow K$, but has high computational cost and poor conditioning for $J \ll K$ [18], [19]. Hence, we next want to explore an approach that is less afflicted by computational cost and poor conditioning.

\section{ChOlesky APPROACH}

\section{A. Cholesky Decomposition}

In Sec. II-C, we have established that the 'smoothness matrix' $\mathbf{C}$ is circulant, which therefore implies a Töplitz structure of $\mathbf{C}$. Thus, we are permitted to write

$$
\mathbf{C}=\left[\begin{array}{ll}
\mathbf{C}_{1} & \mathbf{C}_{2} \\
\mathbf{C}_{2}^{\mathrm{T}} & \mathbf{C}_{4}
\end{array}\right]=\left[\begin{array}{ll}
\mathbf{C}_{4} & \mathbf{C}_{2}^{\mathrm{T}} \\
\mathbf{C}_{2} & \mathbf{C}_{1}
\end{array}\right],
$$

and not affect the overall matrix.

Further, we know that $\mathbf{C}$ is real-valued and positive semidefinite. It therefore admits a Cholesky decomposition $\mathbf{C}=$ $\mathbf{L L} \mathbf{L}^{\mathrm{T}}$, where $\mathbf{L}$ is a lower-left triangular matrix [20], [25]. Partitioning it analogously to the r.h.s. of (18) leads to

$$
\mathbf{L}=\left[\begin{array}{cc}
\mathbf{L}_{4} & \mathbf{0} \\
\mathbf{L}_{2} & \mathbf{L}_{1}
\end{array}\right]
$$

with $\mathbf{L}_{4} \in \mathbb{R}^{(K-J) \times(K-J)}, \mathbf{L}_{2} \in \mathbb{R}^{J \times(K-J)}$, and $\mathbf{L}_{1} \in \mathbb{R}^{J \times J}$. Using (19), we can expand the Cholesky decomposition of (18) such that

$$
\left[\begin{array}{cc}
\mathbf{C}_{4} & \mathbf{C}_{2}^{\mathrm{T}} \\
\mathbf{C}_{2} & \mathbf{C}_{1}
\end{array}\right]=\left[\begin{array}{cc}
\mathbf{L}_{4} \mathbf{L}_{4}^{\mathrm{T}} & \mathbf{L}_{4} \mathbf{L}_{2}^{\mathrm{T}} \\
\mathbf{L}_{2} \mathbf{L}_{4}^{\mathrm{T}} & \mathbf{L}_{2} \mathbf{L}_{2}^{\mathrm{T}}+\mathbf{L}_{1} \mathbf{L}_{1}^{\mathrm{T}}
\end{array}\right]
$$

Thus, the Schur complement $\mathbf{C}_{1}-\mathbf{C}_{2}^{\mathrm{T}} \mathbf{C}_{4}^{-1} \mathbf{C}_{2}$ simplifies to

$$
\begin{aligned}
\mathbf{C}_{1}-\mathbf{C}_{2}^{\mathrm{T}} \mathbf{C}_{4}^{-1} \mathbf{C}_{2}= & \mathbf{L}_{2} \mathbf{L}_{2}^{\mathrm{T}}+\mathbf{L}_{1} \mathbf{L}_{1}^{\mathrm{T}}- \\
& \quad-\mathbf{L}_{2} \mathbf{L}_{4}^{\mathrm{T}}\left(\mathbf{L}_{4} \mathbf{L}_{4}^{\mathrm{T}}\right)^{-1} \mathbf{L}_{4} \mathbf{L}_{2}^{\mathrm{T}} \\
= & \mathbf{L}_{1} \mathbf{L}_{1}^{\mathrm{T}} .
\end{aligned}
$$

The diagonal elements in the Cholesky factor $\mathbf{L}$ are assumed to be ordered. Since $\mathbf{C}$ has a rank of $K-1$, therefore only the last column of $\mathbf{L}$ will be zero. Therefore the $\mathbf{L}_{4}$ will be invertible provided that $J<K$, thus permitting the simplification step in (20). For the case $J=K$ where there are no missing samples, we can simply operate with $\mathbf{C}=\mathbf{L} \mathbf{L}^{\mathrm{T}}=\mathbf{L}_{1} \mathbf{L}_{1}^{\mathrm{T}}$ to weigh the inner product of $\mathbf{f}_{K \mid K}=\mathbf{f}$.

Since the inversion of $\mathbf{L}_{4}$ is not explicitly required in order to work with $\mathbf{L}_{1}$, it suffices to calculate the Cholesky decomposition of $\mathbf{C}$, such that the smoothness metric can be obtained via

$$
\chi_{p}=\mathbf{f}_{J \mid K}^{\mathrm{H}} \mathbf{L}_{1} \mathbf{L}_{1}^{\mathrm{T}} \mathbf{f}_{J \mid K}=\left\|\mathbf{L}_{1}^{\mathrm{T}} \mathbf{f}_{J \mid K}\right\|_{2}^{2},
$$

which therefore does not suffer from the costly matrix inversion and conditioning problems of the Schur approach in Sec. III-B for $J \longrightarrow K$ or the constrained optimisation approach in [18], [19]. 
TABLE I

ERROR IN POWER OF THE $p$ TH DERIVATIVE WHEN BASED ON $K$ SAMPLES OF $F\left(\mathrm{e}^{\mathrm{j} \Omega}\right)$, WITH VALUES STATED IN DECIBEL.

\begin{tabular}{|r||r|r|r|r|r|r|}
\hline \multicolumn{1}{|c||}{$p$} & $K=8$ & $K=9$ & $K=16$ & $K=17$ & $K=32$ & $K=33$ \\
\hline \hline 1 & -309.5 & -312.7 & -311.1 & -312.7 & -307.0 & -312.2 \\
2 & -309.5 & -312.8 & -312.9 & -312.3 & -307.0 & -312.7 \\
4 & -309.5 & -311.5 & -310.7 & -312.7 & -308.2 & -312.2 \\
6 & -309.5 & -312.6 & -310.8 & -312.9 & -307.1 & -312.3 \\
8 & -309.5 & -312.8 & -31.1 & -309.5 & -268.9 & -253.3 \\
10 & -309.5 & -312.7 & -273.4 & -285.9 & -167.6 & -162.8 \\
12 & -309.5 & -312.6 & -209.3 & -217.6 & -72.8 & -61.4 \\
14 & -309.5 & -307.0 & -136.4 & -134.0 & 11.0 & 33.1 \\
16 & -274.9 & -270.1 & -63.7 & -66.6 & 96.6 & 127.1 \\
18 & -231.3 & -227.8 & 11.7 & 13.2 & 217.4 & 218.5 \\
20 & -178.7 & -173.3 & 78.0 & 82.7 & 303.1 & 323.0 \\
\hline
\end{tabular}

\section{B. Simplification via $Q R$}

Since $\mathbf{C}=\mathbf{W D}_{K, L, p}^{2} \mathbf{W}^{\mathrm{H}}$ represents the eigenvalue decomposition of $\mathbf{C}$, we can obtain the Cholesky factor $\mathbf{L}^{\mathrm{T}}$ by means of a QR decomposition of $\mathbf{D}_{K, L, p} \mathbf{W}^{\mathrm{H}}=\mathbf{U R}$, such that $\mathbf{R} \in \mathbb{R}^{K \times K}$ is an upper right triangular matrix and $\mathbf{U} \in \mathbb{C}^{K \times K}$ is unitary. Partitioning this QR decomposition as

$$
\mathbf{D}_{K, L, p} \mathbf{W}^{\mathrm{H}}=\left[\begin{array}{ll}
\mathbf{U}_{1} & \mathbf{U}_{2}
\end{array}\right] \cdot\left[\begin{array}{cc}
\mathbf{R}_{4} & \mathbf{R}_{2} \\
\mathbf{0} & \mathbf{R}_{1}
\end{array}\right],
$$

with $\mathbf{U}_{1} \in \mathbb{C}^{K \times(K-J)}, \mathbf{U}_{2} \in \mathbb{C}^{K \times J}, \mathbf{R}_{4} \in \mathbb{R}^{(K-J) \times(K-J)}$, $\mathbf{R}_{2} \in \mathbb{R}^{(K-J) \times J}$, and $\mathbf{R}_{1} \in \mathbb{R}^{J \times J}$, we obtain $\mathbf{L}_{1} \mathbf{L}_{1}^{\mathrm{T}}=$ $\mathbf{R}_{1}^{\mathrm{T}} \mathbf{U}_{2}^{\mathrm{H}} \mathbf{U}_{2} \mathbf{R}_{1}=\mathbf{R}_{1}^{\mathrm{T}} \mathbf{R}_{1}$ or $\mathbf{L}_{1}^{\mathrm{T}}=\mathbf{R}_{1}$, such that

$$
\chi_{p}=\left\|\mathbf{L}_{1}^{\mathrm{T}} \mathbf{f}_{J \mid K}\right\|_{2}^{2}=\left\|\mathbf{R}_{1} \mathbf{f}_{J \mid K}\right\|_{2}^{2} .
$$

Hence, a Cholesky decomposition of $\mathbf{C}$ is not required, and instead a QR decomposition of $\mathbf{D}_{K, L, p} \mathbf{W}^{\mathrm{H}}$ suffices in order to compute the smoothness associated with a Dirichlet interpolation through a reduced set of $J \leq K$ sample points.

\section{Simulations and Results}

\section{A. Numerical Examples}

We first provide some numerical examples for calculating the power $\chi_{p}$ in a $p$ th derivative using (12). For this, we assume the example function

$$
F\left(\mathrm{e}^{\mathrm{j} \Omega}\right)=1+\frac{1}{\sqrt{2}} \mathrm{e}^{\mathrm{j} \Omega} .
$$

It is straightforward to analyse that the power for every derivative of $F\left(\mathrm{e}^{\mathrm{j} \Omega}\right)$ is $\sigma_{p}^{2}=\frac{1}{2}$ for $p>0$. Based on various number of sample points $K$, and different values of $p$, evaluation of (12) in double floating point precision leads to errors $10 \log _{10}\left|\sigma^{2}-\chi_{p}\right|^{2}$ as shown in Tab. I. The terms $n^{p}$ in (12) can cause numerical problems for larger $p$, particularly when $K$ (and therefore some values of $n$ ) are high. The method works well for lower values of $p$ and $K$, with results to an accuracy in the region of machine accuracy for $p \leq 6$ for the chosen values of $K$. With Tab. I showing errors for $K=\{8,9,16,17,32,33\}$, the approach works equally well for both odd and even $K$.

For the missing samples problem, we operate with $F\left(\mathrm{e}^{\mathrm{j} \Omega}\right)$ in (23), sampled on a uniform grid of $K=8$ sample
TABLE II

POWER IN THE $p$ TH DERIVATIVE OF AN INTERPOLATION THROUGH $J=1 \ldots K$ SAMPLE POINTS OF $F\left(\mathrm{e}^{\mathrm{j} \Omega}\right)$ WITH $K=8$.

\begin{tabular}{|c||c|c|c|c|c|c|c|}
\cline { 2 - 8 } \multicolumn{1}{c|}{} & \multicolumn{7}{c|}{$\chi_{p}$} \\
\hline$J$ & $p=1$ & $p=2$ & $p=3$ & $p=4$ & $p=5$ & $p=7$ & $p=10$ \\
\hline \hline 1 & 0.0000 & 0.0000 & 0.0000 & 0.0000 & 0.0000 & 0.0000 & 0.0000 \\
2 & 0.0921 & 0.1925 & 0.2354 & 0.2465 & 0.2491 & 0.2499 & 0.2500 \\
3 & 0.1825 & 0.3315 & 0.4446 & 0.4859 & 0.4965 & 0.4998 & 0.5000 \\
4 & 0.2827 & 0.4080 & 0.4729 & 0.4931 & 0.4983 & 0.4999 & 0.5000 \\
5 & 0.3773 & 0.4649 & 0.4933 & 0.4990 & 0.4999 & 0.5000 & 0.5000 \\
6 & 0.4468 & 0.4895 & 0.4985 & 0.4998 & 0.5000 & 0.5000 & 0.5000 \\
7 & 0.4886 & 0.4989 & 0.4999 & 0.5000 & 0.5000 & 0.5000 & 0.5000 \\
8 & 0.5000 & 0.5000 & 0.5000 & 0.5000 & 0.5000 & 0.5000 & 0.5000 \\
\hline
\end{tabular}

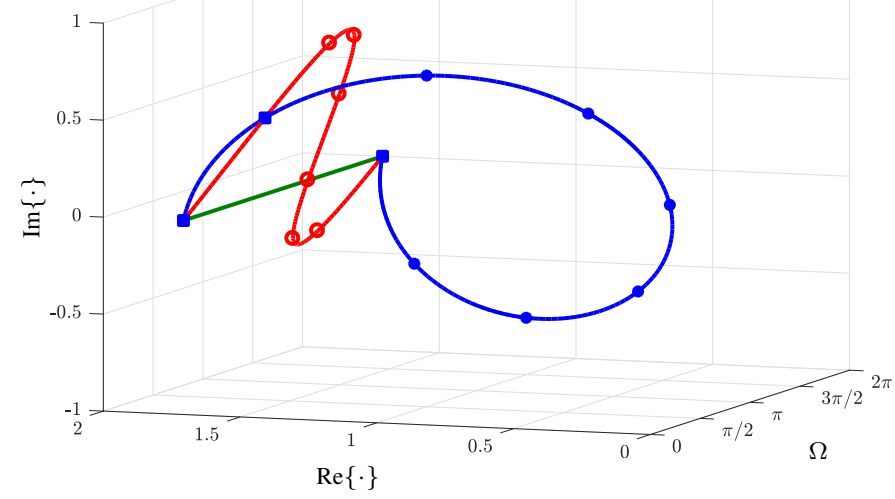

Fig. 1. Example function $F\left(\mathrm{e}^{\mathrm{j} \Omega}\right)$ (blue) and interpolant $\hat{F}\left(\mathrm{e}^{\mathrm{j} \Omega}\right)$ based on $\mathbf{f}_{1 \mid 8}$ (green) and $\mathbf{f}_{2 \mid 8}$ (red), i.e. the first one or two sample points out of $K=8$.

points. However, we base the smoothness cost only on the first $J$ sample points according to Sec. III, with results for $p=\{1,2,3,4,5\}$ summarised in Tab. II. For $J=1$, i.e. a single sample point, the smoothest interpolation is a constant function with zero gradient, hence $\chi_{p}=0$ for $J=1$ and independent of $p$. For $J=2$, it is possible to construct a complex exponential of smaller amplitude through the first two sample points, as indicated in Fig. 1. This complex exponential only oscillates on a line in the complex plane, and differs by $1 / \sqrt{2}$ in amplitude compared to $F\left(\mathrm{e}^{\mathrm{j} \Omega}\right)$. For $J>2, \chi_{p}$ tends to $\sigma^{2}=\frac{1}{2}$ as both $J$ and $p$ increase.

\section{B. Computational Complexity}

To evaluate the smoothness $\chi_{p}$ for a single $\mathbf{f}_{J \mid K}$, i.e. for one instance of $K$ and $J \leq K$, computational complexities in terms of multiply-accumulate operations were derived in [18]. We compare these there to the computational cost of the proposed approach, which requires a $\mathrm{QR}$ decomposition of $\mathbf{D}_{K, L, p} \mathbf{W}^{\mathrm{H}} / \sqrt{K}$, which $\mathcal{O}\left(\frac{1}{3} K^{3}\right)$ multiply-accumulates without exploiting any further structural aspects. These costs are compared for different values of $K$ and ratios $\frac{N}{K}$ in Fig. 2. As detailed earlier, Schur is costly for $N \ll K$, while the constraint optimisation approach solving a minimum variance distortionless response (MVDR) problem is most costly for $J \longrightarrow K$. The proposed approach is independent of $J$, and generally has a computational cost that can always be undercut by either the Schur or MVDR approach. 


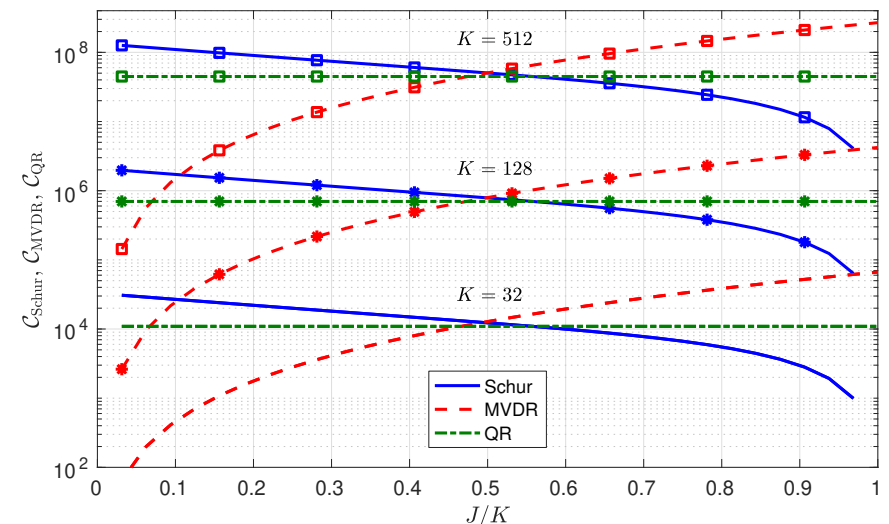

Fig. 2. Cost of a smoothness evaluation for a single instance of $J$ out of $K$ sample points using Schur, MVDR [18], [19] and the proposed approaches.

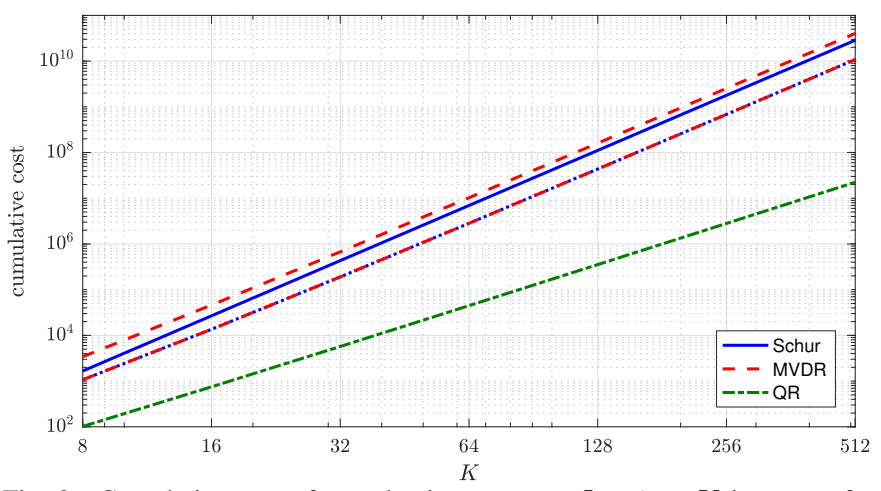

Fig. 3. Cumulative cost of a evaluating $\chi_{p}$ over $J=1 \ldots K$ instances for different values of $K$; comparing the Schur (blue), MVDR (red dashed) [18], [19], and the best combination of Schur/MVDR (red-blue) to the proposed Cholesky/QR approach (green dash-dotted).

For the problem in [16], where for a fixed value of $K$, $J$ goes from one to $K$ to iteratively calculate the cost for a Viterbi-style maximum likelihood sequence estimator, the compared methods behave differently from Fig. 2. While MVDR and Schur approaches require a recalculation of a smoothness metric for every value of $J$, a single QR decomposition suffices for the proposed Cholesky/QR approach. Thus, the cumulative cost in terms of multiply-accumulate operations over all $K$ iterations is given in Fig. 3. The curves for Schur and MVDR approaches are accompanied by a scheme that will, for every value $J$, pick the less expensive of the two, bearing in mind the different dependencies analysed in Fig. 2. All of these however are significantly more expensive than the proposed Cholesky/QR approach.

\section{CONCLuSions}

Based on a trigonometric or Dirichlet interpolation through a potentially incomplete set of sample points of a $2 \pi$-periodic function, we have reviewed how a metric for smoothness - required to extract analytic and therefore infinitely differentiable functions - can be calculated based on the sample points only using the Schur complement of a 'smoothness matrix'. Based on this Schur complement with its inherent matrix inversion. we have evaluated an inversion-free Cholesky decomposition. The latter can be easily calculated via a standard QR decomposition from given quantities. We have demonstrated the accuracy of the proposed approach, and highlighted how the cumulative complexity encountered in an application such as the extraction of analytic eigenvalues from a parahermitian matrix, can be significantly lower than the existing methods.

\section{REFERENCES}

[1] J. G. McWhirter, P. D. Baxter, T. Cooper, S. Redif, and J. Foster, "An EVD algorithm for para-hermitian polynomial matrices," IEEE Trans. Signal Processing, 55(5):2158-2169, May 2007.

[2] S. Weiss, J. Pestana, and I.K. Proudler, "On the existence and uniqueness of the eigenvalue decomposition of a parahermitian matrix," IEEE Trans. Signal Processing, 66(10):2659-2672, May 2018.

[3] S. Weiss, J. Pestana, I.K Proudler, and F.K. Coutts, "Corrections to "On the existence and uniqueness of the eigenvalue decomposition of a parahermitian matrix"," IEEE Trans. Signal Processing, 66(23):63256327, Dec. 2018

[4] S. Redif, J. McWhirter, and S. Weiss, "Design of FIR paraunitary filter banks for subband coding using a polynomial eigenvalue decomposition,” IEEE Trans. Signal Processing, 59(11):5253-5264, Nov. 2011.

[5] S. Redif, S. Weiss, and J.G. McWhirter, "Sequential matrix diagonalization algorithms for polynomial EVD of parahermitian matrices," IEEE Trans. Signal Processing, 63(1):81-89, Jan. 2015.

[6] F.K. Coutts, I.K. Proudler, and S. Weiss, "Efficient implementation of iterative polynomial matrix EVD algorithms exploiting structural redundancy and parallelisation," IEEE Trans. Circuits \& Systems I, 66(12)::4753-4766, Dec. 2019.

[7] S. Redif, S. Weiss, and J.G. McWhirter, "Relevance of polynomial matrix decompositions to broadband blind signal separation," Signal Processing, 134:76-86, May 2017.

[8] M. Alrmah, S. Weiss, and S. Lambotharan, "An extension of the MUSIC algorithm to broadband scenarios using polynomial eigenvalue decomposition," in EUSIPCO, Barcelona, Spain, pp. 629-633, Aug. 2011.

[9] S. Weiss, S. Bendoukha, A. Alzin, F. Coutts, I. Proudler, and J. Chambers, "MVDR broadband beamforming using polynomial matrix techniques," in EUSIPCO, Nice, France, pp. 839-843, Sep. 2015

[10] B. De Moor and S. Boyd, "Analytic properties of singular values and vectors," KU Leuven, Tech. Rep., 1989.

[11] A. Bunse-Gerstner, R. Byers, V. Mehrmann, and N. K. Nicols, "Numerical computation of an analytic singular value decomposition of a matrix valued function," Numer. Math, 60:1-40, 1991.

[12] K. Wright, "Differential equations for the analytic singular value decomposition of a matrix," Numerische Mathematik, 63(1):283-295, Dec. 1992.

[13] L. Dieci and T. Eirola, "On smooth decompositions of matrices," SIAM Journal on Matrix Analysis and Applications, 20(3):800-819, 1999.

[14] D. Janovská, V. Janovský, and K. Tanabe, "An algorithm for computing the analytic singular value decomposition," Int. J. Mathematical and Computational Sciences, 2(11):765-770, 2008.

[15] Y. Nakatsukasa, V. Noferini, and N. Trefethen, "Computing the analytic SVD," Chebfun - numerical computing with functions, 2016.

[16] S. Weiss, I. K. Proudler, F. K. Coutts, and J. Pestana, "Iterative approximation of analytic eigenvalues of a parahermitian matrix EVD," in IEEE ICASSP, Brighton, UK, May 2019.

[17] J. Selva, "FFT interpolation from nonuniform samples lying in a regular grid," IEEE Trans. Signal Processing, 63(11):2826-2834, June 2015.

[18] S. Weiss and M. D. Macleod, "Maximally smooth dirichlet interpolation from complete and incomplete sample points on the unit circle," in IEEE ICASSP, Brighton, UK, May 2019.

[19] S. Weiss, I.K. Proudler, and M.D. Macleod, "Measuring smoothness of real-valued functions defined by sample points on the unit circle," in SSPD, Brighton, UK, May 2019.

[20] G.H. Golub and C.F. Van Loan, Matrix Computations, 3rd ed. Baltimore, Maryland: John Hopkins University Press, 1996.

[21] A.V. Oppenheim, R.W. Schafer, and J.R. Buck, Discrete-Time Signal Processing, 2nd ed. Pearson, 1999.

[22] B. Girod, R. Rabenstein, and A. Stenger, Signals and Systems. Chichester: J. Wiley \& Sons, 2001.

[23] A. Zygmund, Trigonometric Series, 2nd ed. Cambridge Uni. Press, 1959.

[24] W. Wirtinger, "Zur formalen Theorie der Funktionen von mehr komplexen Veränderlichen," Mathematische Annalen, 97:357 - 376, 1926.

[25] I. Bronshtein and K. Semendyayew, Handbook of Mathematics. Heidelberg: Springer, 2015. 